\title{
Ongoing Developments of the Cryo-SEM/STEM Technique
}

\author{
Alan C. Robins ${ }^{1}$ \\ ${ }^{1 .}$ E. A. Fischione Instruments Inc. Export, PA
}

The scanning electron microscope (SEM), with its wide variety of different detectors for imaging or analysis, proves to be an extremely versatile tool for studying the structure of bulk specimens. With the addition of a Scanning Transmission Electron Microscope (STEM) detector, the instrument can also be used for imaging thin sections, aqueous suspensions applied to a TEM-style EM grid, or electron transparent specimens. The entire instrument can be further equipped, for imaging frozen-hydrated bulk samples, with the addition of an on-column or remote cryogenic preparation chamber, a cryo-transfer system, and a nitrogen cooled SEM stage. Low temperature or cryo-SEM techniques have been widely used and adapted as the resolution of the scanning electron microscope has improved [1]. With the introduction of field emission gun technology, a finely focused probe produces sub-nanometer resolution even at low accelerating voltages.

Specimens in solution are either prepared in bulk carriers, such as high pressure freezing planchets, or on EM grids. Cryo-preservation is executed through the use of high-pressure or plunge freezing apparatus. The value of preparing a bulk sample allows for the structural analysis of samples over many tens of microns. A specimen is typically frozen and then transferred to the preparation chamber where it is then fractured, etched and coated prior to examination on a cold stage in the SEM.

Cryo-SEM imaging also benefits from sputtered or evaporated ultra fine grain or amorphous coatings. This is applied to the sample surface to impart conductivity and enhance the visualization of fine structure [2]. In some cases a shadowed primary metallic coating is used which helps enhance contrast while imaging the specimen in backscatter mode. The advantage of the metal coating is that it also reduces beam damage to the specimen.

With the advancement of SEM instrumentation, sample cryo-preservation methods, and metal coating techniques, two branches of cryo-SEM were established. The first includes systems in which all specimen preparation and coating processes are performed remotely. Subsequently, the specimen is then cryo-transferred to the imaging position in the microscope. During transfer the specimen has to be cooled and shielded to stop ice contamination from developing on top of the coating. With in-lens FESEM the remote approach is the only option where a typical cryo transfer holder, used for transmission electron microscopy (TEM), is interfaced to a remote preparation chamber and a goniometer on the microscope [3]. The specimen is cooled in the preparation chamber and microscope by the same liquid nitrogen dewar. Secondly, on-column cryogenic preparation systems have been continually improved to keep up with SEM developments. Care has to be taken to ensure no loss of instrument resolution occurs by incorporating a vibration damped turbo pump. The preparation chamber is attached directly on the column were the sample is fractured, coated and then imaged on a cold stage in the SEM. The SEM stage is cooled to low temperatures, typically $-170^{\circ} \mathrm{C}$, either by continuous flow of cold nitrogen gas or conduction cooling from a dewar attached to the column.

The different types of preparation techniques will be reviewed with associated application images from biology, food, pharmaceuticals and healthcare. 


\section{References:}

[1] P Echlin, Low-Temperature Microscopy and Analysis (Plenum Press, London) 1992, p. 349-411

[2] W Wergin, EF Erbe, and AC Robins, Scanning 15(Supplement III) (1993), p. 71.

[3] RP Apkarian, KL Caran and KA Robinson, Microsc. Microanal. 5 (1999), p. 197-207

[4] ER Wright and VP Conticello, Advanced Drug Delivery Reviews 54 (2002), p. 1057-1073.
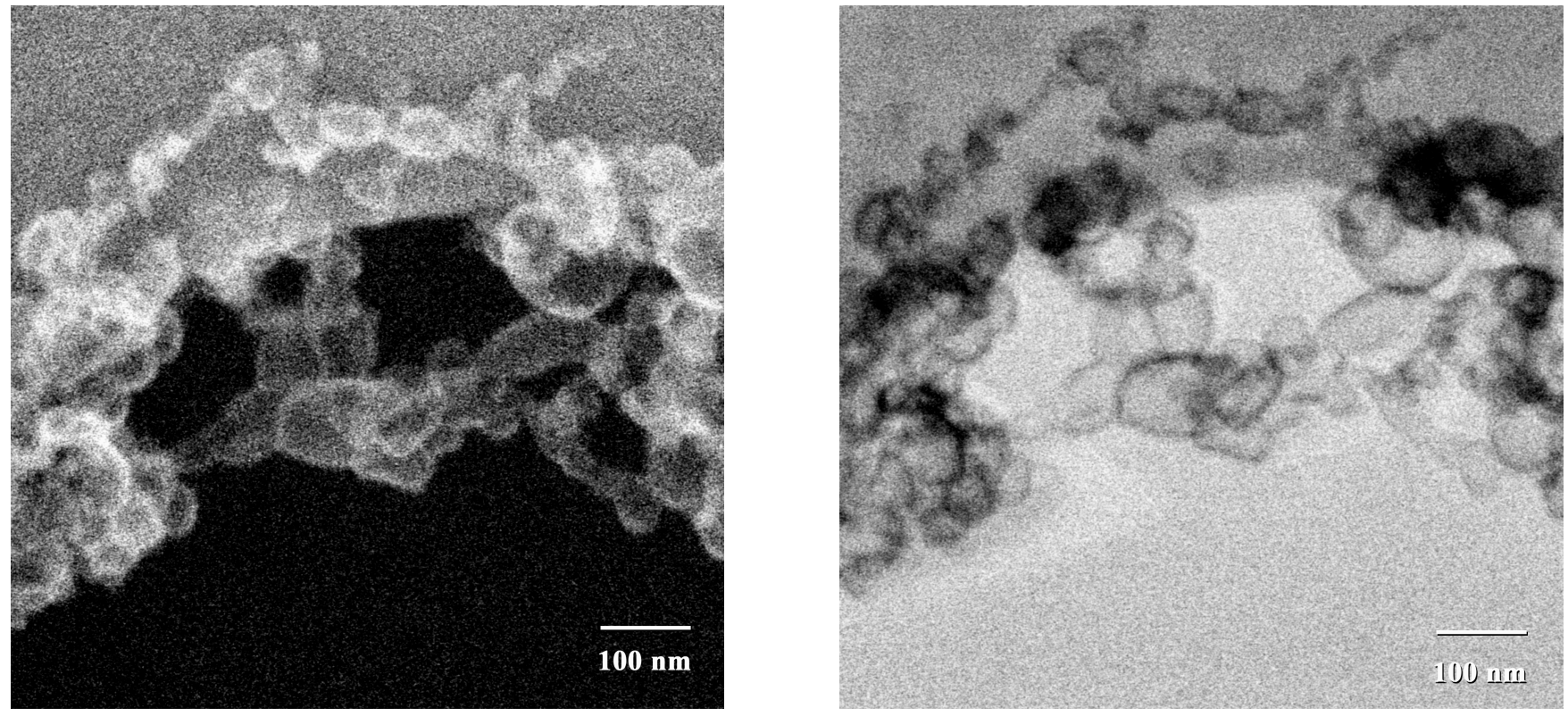

Figure 1. Dilute solution of an elastin-mimetic block copolymer [4], at high magnification, imaged with Cryo-HRSEM (left) and Cryo-HRSTEM (right). 